\title{
Quantification of Cardiac Myocyte-Specific Transcriptional Regulation During Development
}

\author{
Richard L. Goodwin ${ }^{*}$, Ann Marie Harrill, Janea K. Sweet, and Heather J. Evans \\ *University of South Carolina, School of Medicine, Department of Cell and Developmental Biology \\ and Anatomy, Columbia, South Carolina 29209.
}

An intriguing question in developmental biology is how cells with identical genomes differentiate into a wide variety of adult tissues. A viable hypothesis is that differential gene expression (transcription) contributes to regulation of the developmental fate of tissues. We are particularly interested in the gene regulation of developing cardiac myocytes. During the course of normal heart development, cardiac myocytes undergo numerous phenotypic changes as they reach their adult form. One such change is their withdrawal from the cell cycle, transitioning from a proliferative phenotype (hyperplastic) to a cell-growth (hypertrophic) phenotype. When and where myocytes drop out of the cell cycle, and make this transition, plays an important role in both normal and pathogenic morphogenesis of the heart. We have developed a model to investigate the molecular mechanisms that regulate this critical aspect of heart development, a key component of which is a novel collagen tube construct onto which cardiac myocytes are seeded. Embryonically-derived tissues grown on the tube substrate attach, proliferate, and spontaneously contract as a syncytium. Using the tube model, we are investigating the transcriptional regulation of cell cycle genes and markers of differentiation. Other cells types are present in our primary cultures, including endocardial endothelial cells, vascular endothelial cells, vascular smooth muscle cells, and fibroblasts. These non-cardiac myocytes have very different proliferative potentials and therefore interfere with analysis of cardiac myocytes. The development of Laser Capture Microscopy (LCM) provides the opportunity to analyze cardiac myocyte-specific processes. Using LCM technology, cardiac myocytes are isolated from primary cultures by pulling stained cardiac myocytes away from non-cardiac myocytes (figure 1). RNA is then isolated from the 'captured' cardiac myocytes in order to analyze specific transcripts. Using current protocols RNA can be isolated from as many as hundreds of cells or as little as a single cell. Either way small quantities of RNA are obtained by this method. Quantifying the amount of specific transcripts is possible using the highly sensitive real time reverse transcription polymerase chain reaction (real time RT-PCR). Recently, new mathematical models have been developed to accurately quantify specific transcripts [1]. However, quantifying the small amount of starting material obtained from LCM protocols has been problematic. Capillary gel electrophoresis (CE) is capable of quantifying these small quantities RNA and has the potential to over come this technical hurdle. Furthermore, CE can be used to access the quality of the RNA as well. Combining the use of these novel technologies has the potential to delineate the molecular mechanisms that regulate the cell cycle of developing cardiac myocytes. Understanding the mechanisms that regulate cardiac development will set the stage for novel therapies of myocardial repair. 


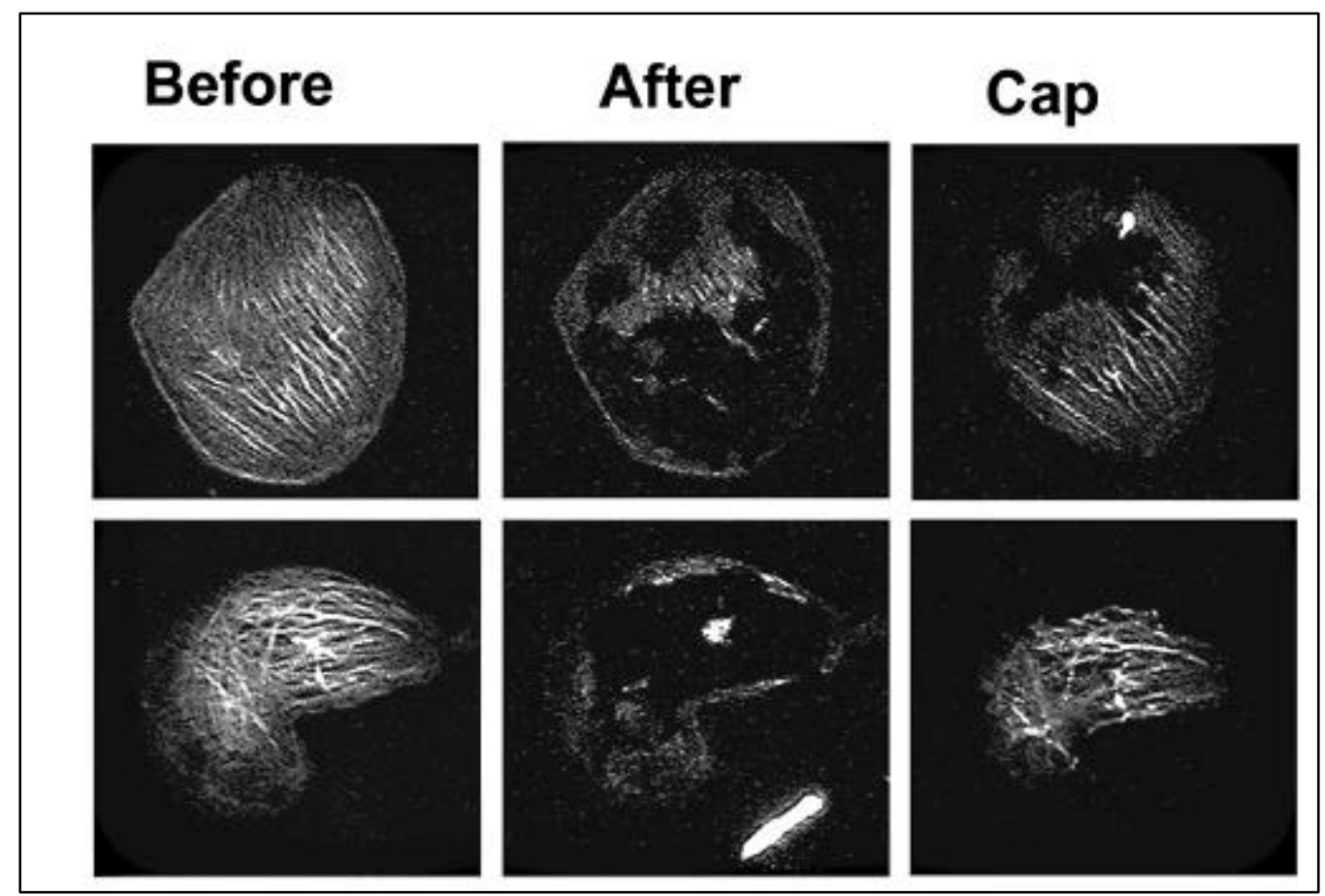

Figure 1. The Use of Laser Capture Microscopy (LCM) to Isolate Muscle Specific Material from Embryonic Heart Primary Cultures. Embryonic day 15 rat cardiac cultures were plated on single well chamber slides coated with fibronectin and grown for five days and stained with the muscle-specific monoclonal antibody, MF-20. Using the Arcturus Pix Cell II Laser capture system, MF-20 positive cells were collected from the primary cultures on CapSure HS high sensitivity LCM transfer film. In the Before column two examples of MF-20 positive cardiac myocytes are shown. The After column shows two examples of cardiac myocytes after the laser capture process. The Cap column shows two examples the material isolated from the cardiac myocytes that has been pulled off the slide by the transfer film cap. RNA is isolated from the cap material and was used for real time RT-PCR analysis.

Reference:

[1] Bustin, SA. Quantification of mRNA using real-time reverse transcription PCR (RTPCR): trends and problems. J Mol Endocrinol. 2002 Aug;29(1):23-39. 